\title{
Heat Conduction in Insulated Metal Roof Decks during Fire: A Computational Approach
}

\author{
D. BREIN and P. G. SEEGER
}

Forschungsstelle für Brandschutztechnik an der Universität Karlsruhe (TH)

Hertzstr. 16, D-7500 Karlsruhe 21, FRG

The heat conduction through lightweight roofs is modeled with melting and decomposition processes involved. The derived set of equations is solved using a numerical difference procedure, which has been enlarged widely for these processes. The computer simulations for several roof assemblies are compared with experimental results from large scale fire tests with flat lightweight roofs exposed to point fire sources underneath the roof. The simulation results show good agreement with data points as to the tendency of the curves The maximum temperatures reached show minor deviations from the test data and give a good estimate for real situations. Differences among the curves are due to scarcely known physical parameters with temperature and perhaps to some simplifying assumptions whose validity will be studied in further work.

\section{INTRODUCTION}

The extent to which lightweight insulated steel deck roofs become involved in a fire, esp. in large industrial premises, made it necessary to carry out large scale fire tests with usual roof assemblies in addition to those tests already mentioned in a literature review by Hofmann/1/. The test program was planned with the intention to study the behaviour of a roof before flash-over would occur in a fire test room. A series of six preliminary tests with relatively small point fire sources had been performed by Brein and Seeger /2/ in 1977. while a subsequent test series of another eleven large scale fires with higher fire loads up to $400 \mathrm{~kg}$ wood cribs was completed at the Fire Research Station in Karlsruhe recently. These fire loads had been concentrated to a sma 11 part of the total area of the fire test room. The final test report by Brein and Seeger /3/ and the conclusions drawn herein have led to proposals for a better design of roof assemblies towards a higher fire safety.

The tests were accompanied by an ad hoc comittee of experts with the intention to have a test program carried out which would satisfy practical needs. A summary of the committee's work is given by Becker et.al. /4/ while an interpretation of the design proposals with exemplary sketches is found in a paper from Federolf $/ 5 /$.

Descriptions of the weather-protected test bullding with a total area of $83 \mathrm{~m}^{2}$ are found in $/ 2,3 /$. The roof assemblies included trapezoidally corrugated 
steel decks of thicknesses $.75 \mathrm{~mm}$ to $1.13 \mathrm{~mm}$ supported by steel beams $4 \mathrm{~m}$ above floor. These decks were covered by bituminous vapour barriers (which were sometimes omitted) and several types of combustible and non-combustible insulations of thicknesses ranging from 40 to $70 \mathrm{~mm}$, depending on the individual thermal conductivity $k$ of the tested specimen. The reason for a choice of several thicknesses was, that the product of the individual $k$ times the thickness of the relevant insulation board should be kept constant and equal to that of expanded polystyrene foam with a bulk density of $20 \mathrm{~kg} / \mathrm{m}^{3}$ and $50 \mathrm{~mm}$ thickness. The insulation boards were then covered with two layers of bituminous roofing membranes according to technical rules. Further on in this paper the cerm "roof assembly" is used for the compound above the steel deck.

The tests have shown, that there are two main influences on the fire behaviour of lightweight roofs: the one is the construction itself, which comprises the type of fastening the decks at the side laps with each other and upon the supporting members or the design at the perimeter of the roof. As an example of the provisions made for a late collapse of the steel deck we have used rivets with a high melting point to fasten the steel decks to each other along the laps. The other main influence with respect to the time-dependent increase of surface temperature is the mechanical response of the insulation boards and of the roofing membranes to the developing fire underneath the roof since thin layers with relatively high thermal conductivity will lead to a faster increase in surface temperature than will thick layers with a relatively low thermal conductivity and so support ignition in an early stage of the fire.

Some insulations will melt at low temperatures of about $100^{\circ} \mathrm{C}$ like expanded polystyrene foam, others will keep their shape up to very high temperatures of near $800^{\circ} \mathrm{C}$ or more like mineral wool, others will show a behaviour like urethane rigid foam which pyrolyses and cracks reducing its thickness continuously while the fire is going on underneath.

The results of the fire test series mentioned in $/ 2,3 /$ are very interesting but unsatisfactory as to the interpretation of the results for roofs with thicker insulations as prescribed by recent technical and administrative rules towards higher energy savings. Consequently, to diminish the costs for research on the fire behaviour of lightweight roofs, a research program has been initiated to calculate the time-dependent heat conduction through lightweight roofs with several types of insulation boards while exposed to a fire source of restricted area underneath. This work is being partially supported by the "Stiftung Volkswagenwerk", a foundation of a motor-car factory in the Federal Republic of Germany.

\section{DEF INITION OF THE PROBLEM}

The calculation of heat conduction through a lightweight roof exposed to fire from beneath firstly requires several simplifying assumptions concerning the governing mechanisms of heat transfer to and from the roof, i.e. the boundary conditions must be adequately set but such, that they can be handled by a computer program within a reasonable period of time.

In a real fire situation, the roof is being exposed to open flames or to the fire plume from combustion of $\mathrm{e} . \mathrm{g}$. stored goods. In the early stages of a fire, the main mode of heat transfer to the lower surface of the roof will be only by convection but in case. that the fire has grown, radiative transfer from open flames will perhaps become the dominant mode. The flames and hot gases impinging on the lower surface of the celling are being bent to the horizontal direction and flow out of the fire test room at a distance from the 
fire origin. It should be mentioned, that no ventilation openings had been installed in the tests referred to above and that the only opening was at the front side of the test room.

Heat passes through the steel deck by conduction. Depending on which cross-section of the roof is being considered, heat is then transferred to the lowest layer of the roof assembly by direct contact via the flanges or by radiation and by convection of horizontally flowing gases of decomposition inside the roof assembly via the ribs to that part of the lowest layer of the roof assembly, which is placed over the rib openings. The former heat conduction through the several layers of the roof assembly can be evaluated, if the melting mechanisms of the vapour barrier and the bituminous roofing merbranes can be modeled. In addition the processes taking place as the insulations are being thermally cracked or molten have to be simulated. The processes considered lead to a reduction in shape and thickness and may also result in a partial outflow of molten products into the rib section of the steel deck if either no vapour barrier with a mechanically strong reinforcement is in use or if no vapour barrier is used at all. At the upper surface of the roof, radiation may be neglected as long as the temperatures are considerably lower than the ignition temperature of the roofing membranes. In this case, heat transfer by free convection has to be considered as the only heat transfer mechanism.

A lot of time-dependent temperature curves at several positions in the roofs has been measured and a lot of other data is avallable from the fire tests for comparison. But there is still a lack of knowledge about the dependence of physical parameters like the thermal conductivity $\mathrm{k}$ with temperature for most of the building materials dealt with in this paper. Therefore the values used here are approximations only on purpose to compare experimental with computed data. The reason for missing data on physical values at high temperatures is clear for materials commonly in use at room temperature.

\section{THE MATHEMATICAL MODEL}

\section{Heat Conduction and Boundary Conditions}

The mathematical model consists of the parabolic, partial-differential equation for the time-dependent heat conduction in solids, the relevant modeled equations for melting and thermal degradation processes and the boundary conditions at the lower and the upper surfaces of the roof assembly, respectively. The conventional "Fourier"- equation models the heat balance of a solid volume element. In the form used in this work, the equations that constitute the mathematical model are written as follows:

$\frac{\partial}{\partial x}\left[k(T) \frac{\partial T}{\partial x}\right]+\frac{\partial}{\partial y}\left[k(T) \frac{\partial T}{\partial y}\right]+W=\rho c \frac{\partial T}{\partial t}$.

This is the two-dimensional heat flow equation, which has no closed-form solution because of the temperature dependence of the thermal properties of the materials used and also because of a nonlinearity of the boundary conditions. Among the schemes available to solve equation (1) on a computer numerically it was transformed into a set of difference equations. The well-known solution scheme will be described in short later in this paper.

The initial and boundary conditions are deduced from the practical problem. As an initial condition a unique temperature distribution was chosen in the roof assembly as a whole and equal to the constant ambient temperature in the test room before the ignition of the fire load. 
The melting and degradation processes with variation of thickness of the insulation boards are modeled for one dimension in space. Consequently the description of boundary conditions is restricted to the (vertical) y-direction of the coordinate system. The boundary conditions at the lower surface of the roof depend on the roof section which is to be considered, i.e. either the region above the flanges or above the ribs of the steel deck. The first case comprises the derivation of a total heat exchange coefficient for both convective and radiative transfer to the steel deck and direct contact between the flange of the steel deck and the roof assembly. The second case comprises both modes below the steel deck and both modes between the ribs and the lower surface of the roof assembly as we 11 .

For a comparison between the experiments and the computer results a thorough study of the heat transfer underneath the steel deck can be omitted because of test data avallable in the flange as well as in the $\mathrm{rib}$ region. The time-dependent temperatures measured are used as input values for the following boundary conditions at the lower surface of the roof assemblies, i.e. at the vapour barrier and at the insulation board, respectively:

Given the steel deck flange temperature, then

$T_{S}=T_{f I a}(t)$

Given the temperature in the cavity of the ribs, then

$h\left(T_{g}(t)-T_{s}\right)=-k\left[\frac{\partial T}{\partial y}\right]_{s}$.

The total heat transfer coefficient $h$ in eq. (3) is the sum of the convective and the radiative heat transfer coefficients. The latter is evaluated using a zone method, see e.g. Hottel and Sarofim $/ 6 /$.

At the interfaces between several layers of the roof assembly no special considerations are needed. This arises from the fact, that

$-k_{1}\left[\frac{\partial T}{\partial y}\right]_{1}=-k_{2}\left[\frac{\partial T}{\partial y}\right]_{2}$.

At the upper side of the roof the boundacy condition is given by an equation similar to eq. (3) with the restrictions, that only convection needs to be considered and that the ambient temperature is constant throughout the test simulation:

$-h\left(T_{s}(t)-T_{a}\right)=k\left[\frac{\partial T}{\partial y}\right]_{s}$.

\section{The Melting Process}

The melting process is modeled for the one dimensional problem. The consumption of heat of fusion is a characteristic of melting processes and if the materials are chemically pure ones, the other characteristic is a constant melting point at the solid-fluid boundary. Considering the materials used for lightweight roofs like bituminous roofing membranes or thermosetting expanded polystyrene foam (EPS), the assumption of a fixed melting point is a strongly simplifying assumption. Looking at the relevant roofing membranes a broad melting region will be found caused by the large range of hydrocarbons asphalt is consisting of. In the real 
situation there arises an adequate difficulty with EPS which will firstly sinter forming cavities and then melt. Modeling of such processes is difficult and therefore as a first approximation the materials were assumed as pure with fixed melting points and a fixed heat of fusion. A reason for this simplification is the fact, that melting processes during real fires will often take place in a small time interval relative to the total duration of the test and so a too exact analysis would have been outside the scope of this work.

The heat input from one side will result in a continuously advancing phase boundary between the region still solid and the region already fluid of the specimen. Using a heat balance at the boundary, an expression for the phase boundary propagation velocity can be derived. As a simplifying assumption convection flows within the molten materials are assumed negligible because of their small thickness. The phase boundary itself is unable to store heat which leads to the following heat balance:

$q_{\text {in }}=q_{\text {out }}+q_{c}$.

The terms in eq. (5) for input, output and consumption, respectively, are as defined in equations (6) to (8):

$$
\begin{aligned}
& q_{\text {in }}=-k_{f 1}\left[\frac{\partial T}{\partial y}\right]_{p h, f 1}, \\
& q_{\text {out }}=-k_{\text {so }}\left[\frac{\partial T}{\partial y}\right]_{p h, s o}, \\
& q_{c}=H_{f} \rho_{\text {so }}\left[\frac{d y}{d t}\right]
\end{aligned}
$$

Combining equations (6) to (8) with eq. (5) results in an expression for the phase boundary propagation velocity, which is a velocity term relative to the solid region of the material:

$\frac{\mathrm{dy}_{\text {so }}}{\mathrm{dt}}=\frac{1}{\mathrm{H}_{\mathrm{f}} \rho_{\mathrm{so}}}\left[\mathrm{k}_{\mathrm{so}}\left[\frac{\partial \mathrm{T}}{\partial \mathrm{y}}\right]_{\mathrm{ph}, \mathrm{so}}-\mathrm{k}_{\mathrm{f} 1}\left[\frac{\partial \mathrm{T}}{\partial \mathrm{y}}\right]_{\mathrm{ph}, \mathrm{fl}}\right]$.

In case of a material with a great difference in the densities of the solid and the fluid phases. respectively, the increase of the fluid phase is given by the following equation (10) :

$\frac{d y_{f 1}}{d t}=\frac{\rho_{\text {so }}}{\rho_{f 1}} \frac{d y_{s o}}{d t}$

A typical example is EPS. Where the density of the fluid phase is about 50 times the density of the solid phase.

Decomposition Kinetics

The pyrolysis and cracking of insulations of the urethane rigid foam type was modeled in a conventional manner:

$-\frac{d m}{d t}=\left(m-m_{f i}(T)\right) v(T) \quad$ 
The temperature dependent reaction rate constant was defined as an Arrhenius-type equation of first order:

$v(T)=f \exp (-E /(R T))$

With the dimensionless mass loss defined as

$u=\frac{m_{0}-m}{m_{0}-m_{f i, m i n}}$,

and the specific mass. loss in case of a fully decomposed material as

$\mu(T)=\frac{m_{0}-m_{f i}(T)}{m_{0}}$,

and by combining equations (11) to (14) we derive the time-dependent dimensionless mass loss rate as

$\frac{d u}{d t}=\left[\frac{\mu(T)}{\mu_{\max }}-u\right] v(T)$.

Using eq. (15) leads to a heat sink term by pyrolytic reaction which is to be substituted in eq. (1):

$W=-\frac{d u}{d t}{ }_{r}^{H} \rho_{0} \frac{V_{o}}{V_{\text {act }}}$.

The ratio of the volumes in eq. (16) results from the definition of the heat sink as per actual volume whilst the heat of the decomposition reaction is related to the initial mass element before pyrolysis occurs.

In case of a one dimensional analysis a change in volume and thickness respectively can be easily achieved. With the mass of a volume element defined as

$\mathrm{m}=\rho \Delta \mathrm{x} \Delta \mathrm{y} \Delta \mathrm{z}$,

and if a change of thickness is restricted to one dimension of the coordinate system, e.g. to the $y$-direction, the specific thickness can be expressed in terms of the mass and density ratios:

$\frac{\Delta y}{\Delta y_{o}}=\frac{m(u) / m_{o}}{\rho(u) / \rho_{o}}$.

The mass is a function of the dimensionless mass loss per definition. The density value depends on the dimensionless mass loss as well and may be defined in terms of a linear expression:

$\rho(u)=(1-u) \rho_{o}+u \rho_{f l}$. 
The numerical solution of eq. (1) with the boundary conditions and the expressions for the melting and degradation processes described above was performed with the Crank-Nicholson scheme for one dimensional appliances and with the PeacefordRachman scheme in case of two dimensions. The mathematical schemes were adopted from the literature, see e.g. Marsa $1 / 7 /$.

A short description of the solution procedure is concentrated upon the following remarks: using the difference scheme, the continuous governing differential eq. (1) is converted to a set of linear algebraic equations for each time step. This set of linear equations is formed by superposing a grid to the material or compound material in question and then by deriving the first and second order derivatives of the temperature values at the discrete set of grid points. The grid points need not to have a constant distance between each other. The distance is chosen variable according to the necessities of stability of the numerical solution. The set of equations thus derived forms a tridiagonal coefficient matrix which can be solved using a simple, well-known mathematical algorithm. The solution of the matrix leads to an array of temperature values and - in case of pyrolysis reactions - to an array of density values at each node of the grid. These are in turn used for the new difference quotients for a new time step and the resulting set of equations is solved as before.

In case of incorporated melting processes we have included an automatic forward time-step size such, that it can be diminished as soon as the melting point is reached. By this we avoid melting of the whole layer of material within one time step. which would bring down the accuracy of the computations. Detalls of the time-step size choice are beyond the scope of this paper. A full description is given by Brein $/ 8 /$.

\section{RESULTS}

The comparison of selected experimental results with the corresponding computer simulations is illustrated in Figs. 1-3. In each of these figures the computer predictions are represented as broken lines, and the experimental results as continuous curves with symbols. The experimental curves are from the test report mentioned above /3/. The position ' $F$ ', where the temperatures had been measured using themocouples, represents a region of the roof exactly above the fire source with the highest temperatures reached during the tests.

The computer simulations were carried out using the one dimensional approach for heat conduction through a roof assembly in the vertical direction. Horizontal gradients were neglected because of the ratio of vertical to horizontal spacing. Besides, the computations were restricted to one dimension, because melting processes with flow at of material into the rib region of the roof were modeled for this case only. The same is true for pyrolytic processes, where load bearing roof insulations will shrink in vertical direction mainly.

For the computer simulations the following assumptions were used:

- The boundary condition at the lower side of the roof assembly is the timedependent heat flux at the position of thermocouple 'F2' in the cavity of the ribs. Above the upper side of the roof the ambient temperature of the relevant test date is chosen.

- Radiative transfer of heat from the gases within the rib region of the steel deck is neglected. The heat transfer from the ribs at temperature 'F2' to the 
roof assembly due to radiation is derived using the zone method referred to above (see /5/).

- There is no heat loss of the gases within the rib section due to convective flows in horizontal direction.

Fig. I shows the comparison of a mineral-fibre board experiment with the simulattion. In addition to the assumptions described above, the water vapour transfer through the insulation board was neglected. Flowing out of $50 \%$ of the molten part of the vapour barrier into the rib section was allowed. The trend of the computed curves is towards the real (measured) situation, but there are still differences among the curves, which can be explained with roughly approximated physical values, and perhaps with influences, which arise from the positioning of the termocouples during the tests or from water vapour in the mineral-fibre boards. The possible reasons are numerous and it seems difficult to weigh them adequately without thorough studies of each.

Fig. 2 shows the comparison of the EPS test with the computer simulation. With the real behaviour of an expanded polystyrene foam and the approximation of assuming a pure material in mind, the simulation result is rather good. The authors suggest, that the differences might mostly be due to a fixed thermocouple in the test and the formation of cavities within the insulation board, which might lead to questionable data. Another reason is supposed to be unreliable physical values at higher temperatures.

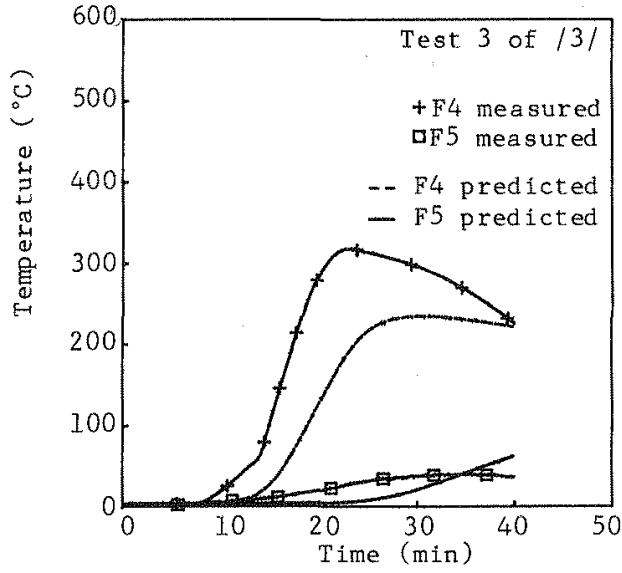

FIGURE 1. Mineral fibre insulation with vapour barrier.

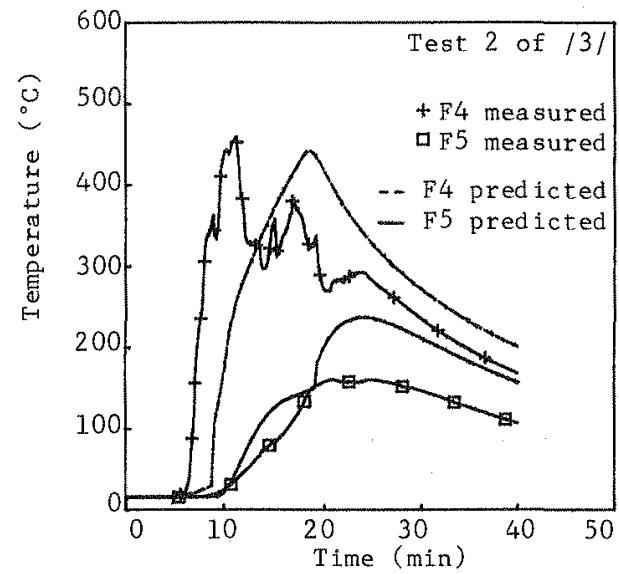

FIGURE 2. Expanded polystyrene foam with vapour barrier.

Fig. 3 shows the comparison of the urethane rigid foam test without vapour barrier with the simulation. Some assumptions concerning the kinetic parameters were incorporated in the simulations. These data were derived from small scale experiments carried out in a laboratory oven to determine the Arrhenius-rate constant, the activation energy and the final density. The heat of the decomposition reaction was evaluated using the formation enthalpies of plausible pyrolysis reactions. Fig. 3 shows a fairly good agreement between test and simulation, esp. with respect to the highest temperature value reached in the midst of the insulation board (thermocouple 'F4'). But there are still differences as to the time scale.

Fig. 4 shows the variation of the specific mass loss with vertical distance with the time as parameter and thus demonstrates the degree of pyrolysis of the foam. 


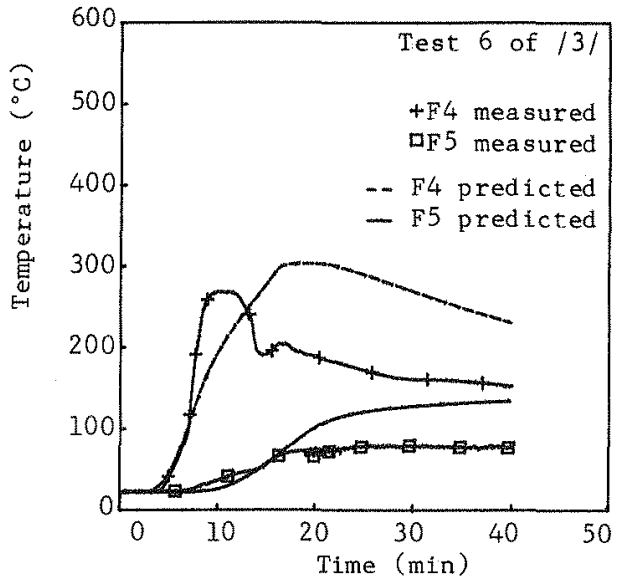

FIGURE 3. Urethane rigid foam, no vapour barrier.

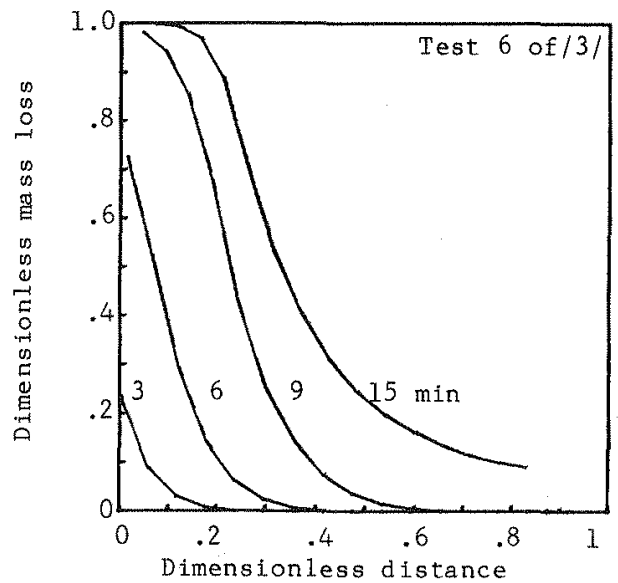

FIGURE 4. Variation of mass loss with distance in the PU board.

\section{SUMMARY}

The heat conduction through lightweight roofs is modeled with melting and decomposition processes involved. The derived set of equations is solved using a numerical difference procedure, which has been enlarged widely for these processes. The computer simulations for several roof assemblies are compared with experimental results from large scale fire tests with flat lightweight roofs exposed to point fire sources underneath the roof. The simulation results show good agreement with data points as to the tendency of the curves. The maximum temperatures reached show minor deviations from the test data and give a good estimate for real situations. Differences among the curves are due to scarcely known physical parameters with temperature and perhaps to some simplifying assumptions, whose validity will be studied in further work.

\section{NOMENC LATURE}

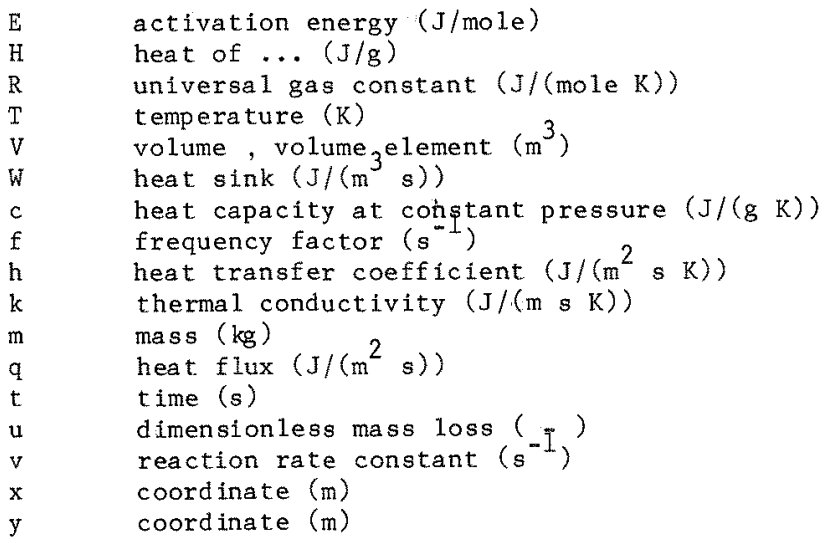




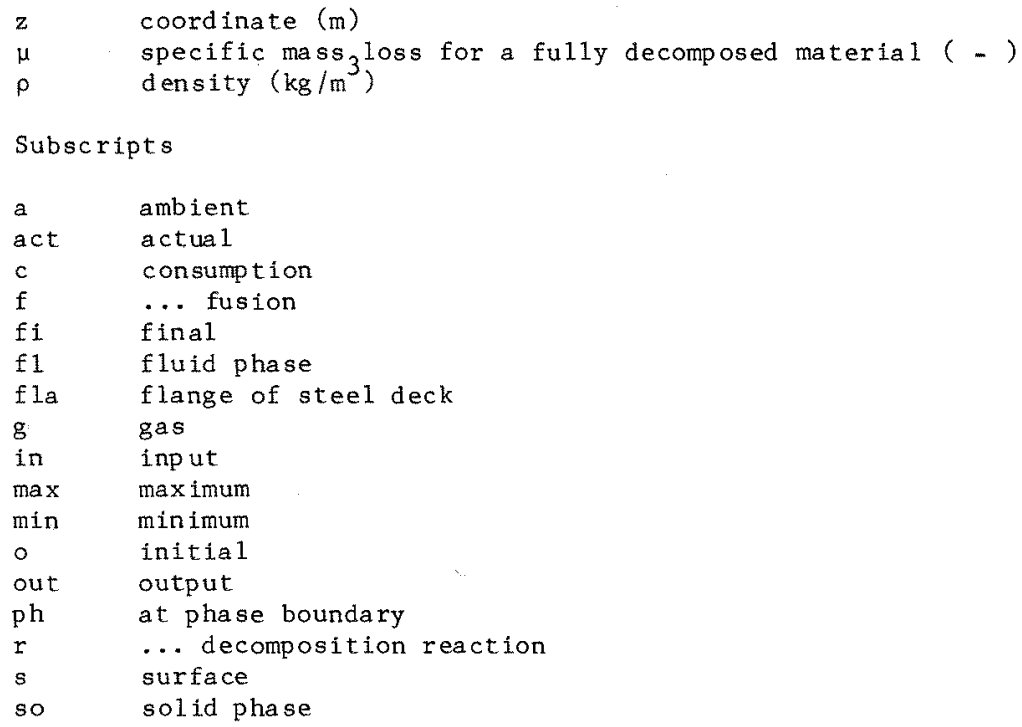

\section{REFER ENCES}

1. Hofmann, K., VFDB-Z 27, 4(1978).

2. Brein,D. and Seeger,P.G., Fire and Materials, Vol. 3, No.3, 1979.

3. Brein,D. and Seeger,P.G., Brandversuche an wärmegedämmten Stahltrapezprofi1dächern, Forschungsstelle für Brandschutztechnik, Karlsruhe (1982).

4. Becker,W. et. a1., VFDB - Z 33,2(1984).

5. Federolf, S. WFB - Z 33,2(1984).

6. Hottel.H.C. and Sarofim,A.F., Radiative Transfer, McGraw-Hi11 (1967).

7. Marsa1.D., Die numerische Lösung partieller Differentialgleichungen, Bibliographisches Institut AG, Zirich (1976).

8. Brein. D.: Final report in preparation (1985). 